\title{
Factors Affecting Coaching Excellence: Qualitative Analysis of the Core Elements of Coaching Ladder
}

\author{
Katsuro Kitamura*, Dexia Yin** \\ Nihon University*, Tohoku University**
}

\begin{abstract}
The purpose of this study was to explore the developmental process of perceptions of coaching expertise of expert professional coaches in Japan, and to identify the factors behind the growth. Eighteen expert coaches served as participants for this study (swimming, football, rowing, athletics, gymnastics, speed skating, basketball, softball, bobsled and table tennis). In-depth, open-ended, semi-structured interviews were conducted with each coach. All interviews were conducted face-to-face by the same researcher who was trained in qualitative techniques. Interviews ranged between 60 to 90 minutes, were recorded with the permission of the participants. The interviews were systematically transcribed verbatim from the IC recorder immediately after the completion of each interview. The data was decontextualized using an inductive procedure for analyzing unstructured qualitative data.

The total number of meaning units related to the contextual component in this study was 287 gathered from eighteen coaches. The inductive analysis process resulted in regrouping these interview transcripts into three categories, 1) aspiration for a change in consciousness through the experience repeated setbacks, 2) deepen the perception of the reconstruction of an intimately relationship, 3) Sophistication of essential and comprehensive practical knowledge.

Although the developmental patterns of coaching expertise were influenced by many factors, this study found significant agreement between the perceptions of the concepts on how expert coaches evaluated their daily activities in relation to athlete's performance development and how the role of reflection affects the development of coaching expertise. 234 Professional development, coaching expertise, interviewing, practical knowledge, reflection.
\end{abstract}

Keywords: professional development; coaching expertise; interviewing; practical knowledge;

reflection. 